\title{
Enucleation with Peripheral Ostectomy and Chemical Cauterization for Unicystic Ameloblastoma and Odontogenic Keratocyst: Primary Modality of Choice or still a Secondary Alternative? A Report of 18 Cases
}

\author{
${ }^{1}$ Tejraj P Kale, ${ }^{2}$ Lingaraj J Ballihalimath, ${ }^{3}$ Saurabh Sharma, ${ }^{4}$ Sharadindu M Kotrashetti
}

\begin{abstract}
Aim: The treatment of odontogenic keratocyst (OKC) and unicystic ameloblastoma (UA) remains controversial. With modern innovations, trend is shifting toward minimally invasive surgical procedures. The aim of this study was to report the outcome of our conservative treatment protocol for OKC and UA, particularly highlighting the effectiveness of enucleation and peripheral ostectomy with chemical cauterization using Carnoy's solution compared with aggressive techniques.
\end{abstract}

Materials and methods: Eight patients with UA and 10 with keratocystic odontogenic tumor (KCOT) who reported to the KLE Vishwanath Katti Institute of Dental Sciences (KLE VKIDS), Belgaum, India, between 2008 and 2015 were included in the study. In addition to clinical and radiographic examination, incisional biopsy was done to confirm the diagnosis. All the patients were treated using enucleation with peripheral ostectomy and application of Carnoy's solution for 5 minutes as a primary modality of choice postoperatively; patients with extensive lesion received an intermaxillary fixation for 5 to 6 weeks. Surgical obturator to obliterate the pathological defect was given in large defects.

Results: The mean length of follow-up was 76 months since the last operation. In our experience, no recurrence has been encountered until May 2017.

Conclusion: Our study suggests that conservative treatment with adequate postoperative follow-up yields clinically acceptable results with less morbidity. However, the patients must adhere to close follow-ups. Our algorithm for managing OKCs and UA of the jaws is also presented. This technique shows comparable results to other more aggressive techniques and can be used especially in large lesions that when treated with resection will interrupt continuity of the jaw.

Clinical significance: For a long time, KCOT and UA were treated aggressively, i.e., through resection, but our study shows conservative treatment holds good. It also reduces the risks and complications associated with surgical resection.

Keywords: Ameloblastoma, Carnoy's, Conservative, Current, Cyst, Keratocyst.

How to cite this article: Kale TP, Ballihalimath LJ, Sharma S, Kotrashetti SM. Enucleation with Peripheral Ostectomy and

${ }^{1-4}$ Department of Oral and Maxillofacial Surgery, KLE VK Institute of Dental Sciences, Belagavi, Karnataka, India

Corresponding Author: Saurabh Sharma, Department of Oral and Maxillofacial Surgery, KLE VK Institute of Dental Sciences Belagavi, Karnataka, India, Phone: +919990041437, e-mail: doc.saurabh@outlook.com
Chemical Cauterization for Unicystic Ameloblastoma and Odontogenic Keratocyst: Primary Modality of Choice or still a Secondary Alternative? A Report of 18 Cases. World J Dent 2017;8(6):467-470.

\section{Source of support: Nil}

Conflict of interest: None

\section{INTRODUCTION}

Unicystic ameloblastoma was first described by Robinson and Martinez ${ }^{1}$ as a cystic lesion that showed clinicoradiographic or gross features of an odontogenic cyst but showed atypical ameloblastomatous epithelial lining with or without luminal or mural tumor growth histopathologically.

Unicystic ameloblastoma in early stage grows slowly with minimal signs and symptoms but later might cause destruction, root resorption, and mobility of teeth in involved region. ${ }^{2}$ It is believed to be less aggressive than the solid or multicystic type of ameloblastoma. ${ }^{3}$

Keratocystic odontogenic tumor stands out of other odontogenic cyst/lesion in the sense of its characteristic histopathological appearance and aggressive behavior, i.e., high recurrence rate. The KCOT accounts for approximately 12 to $14 \%$ of all odontogenic cysts with male predominance and predilection for the posterior mandible. Radiographically, KCOT may appear as unilocular or multilocular radiolucency, with smooth or scalloped margins and sclerotic border. Microscopically, it is characterized by stratified squamous epithelium, i.e., 8 to 10 cell layers in thickness with polarized basal cell layer and a connective tissue wall. ${ }^{4,5}$ Odontogenic keratocyst is of two types: Orthokeratocyst and parakeratocyst, the former being less aggressive and latter showing local destruction with extension into adjacent tissues and a higher rate of recurrence. ${ }^{6}$

Its aggressive nature due to the thin friable wall which is often difficult to enucleate from the bone in one piece and has small satellite cysts within the fibrous wall.

Various treatment modalities for UA and OKC have been documented in literature; however, universally accepted modality is still undecided. Treatment ranges from conservative methods, such as enucleation, decompression, 
marsupialization, and peripheral ostectomy to aggressive treatments which include resection. All techniques have similar goals: Removal of lesion and decreasing chances of recurrence and surgical and functional morbidity.

This article aims at highlighting enucleation and peripheral ostectomy followed by chemical cauterization with Carnoy's solution as an effective treatment modality in cases of UA and KCOT.

\section{MATERIALS AND METHODS}

Eight patients with UA and 10 with KCOT who reported to the Department of Oral and Maxillofacial Surgery, VKIDS, KLE, Belgaum, India, between 2008 and 2015 were included in the study. Clinical and radiographic examination (orthopantomogram) along with incisional biopsy was done to confirm the diagnosis of UA or KCOT. Consent was taken after explaining about the treatment modality and the chance of recurrence.

A treatment plan of enucleation and peripheral ostectomy with chemical cauterization using Carnoy's solution was finalized for all the cases even where the lesion was close to the lower border of the mandible or perforating one of the cortices or causing root resorption of the involved teeth.

Surgery was performed under general anesthesia, mucosal flaps were raised, and buccal decortication was done to gain access to the lesion. Entire lesion was enucleated and peripheral ostectomy was carried out until the healthy bleeding bone was encountered all around. Gauze soaked in Carnoy's solution was packed into the cavity and left in situ for 5 minutes. Cavity was thoroughly irrigated with normal saline, and elastomeric base impressions were taken for fabrication of surgical obturator. Ribbon gauze soaked in sofradex (Framycetin sulfate, Gramicidin) was packed into the cavity, and temporary closure with stay sutures was done. Obturator was given after 48 hours postoperatively filling two-thirds of the defect. It was decided that if any recurrence was seen, same procedure has to be repeated as the pathology is same.

\section{RESULTS}

In our experience, no recurrence has been encountered until May 2017. Care has to be taken while applying the solution to the tissue. Sterile gauze is adapted in the surgical bed, and then gauze soaked in Carnoy's solution is placed in the pathological cavity for 5 minutes taking care not to damage the surrounding soft tissues. During this surgery, reflection of the tissues should be done carefully, and conspicuous irrigation with saline has to be performed after the removal of the gauze with constant aspiration. We evaluated 10 cases of $\mathrm{OKC}$ and 8 cases of UA (Figs 1 and 2) with age between 20 and 45 years (mean: 36 years). Mandible was involved more than maxilla, with a marked occurrence in the posterior mandible. Twelve cases were in tooth-bearing areas and 7 caused dental root resorption. The radiographic finding
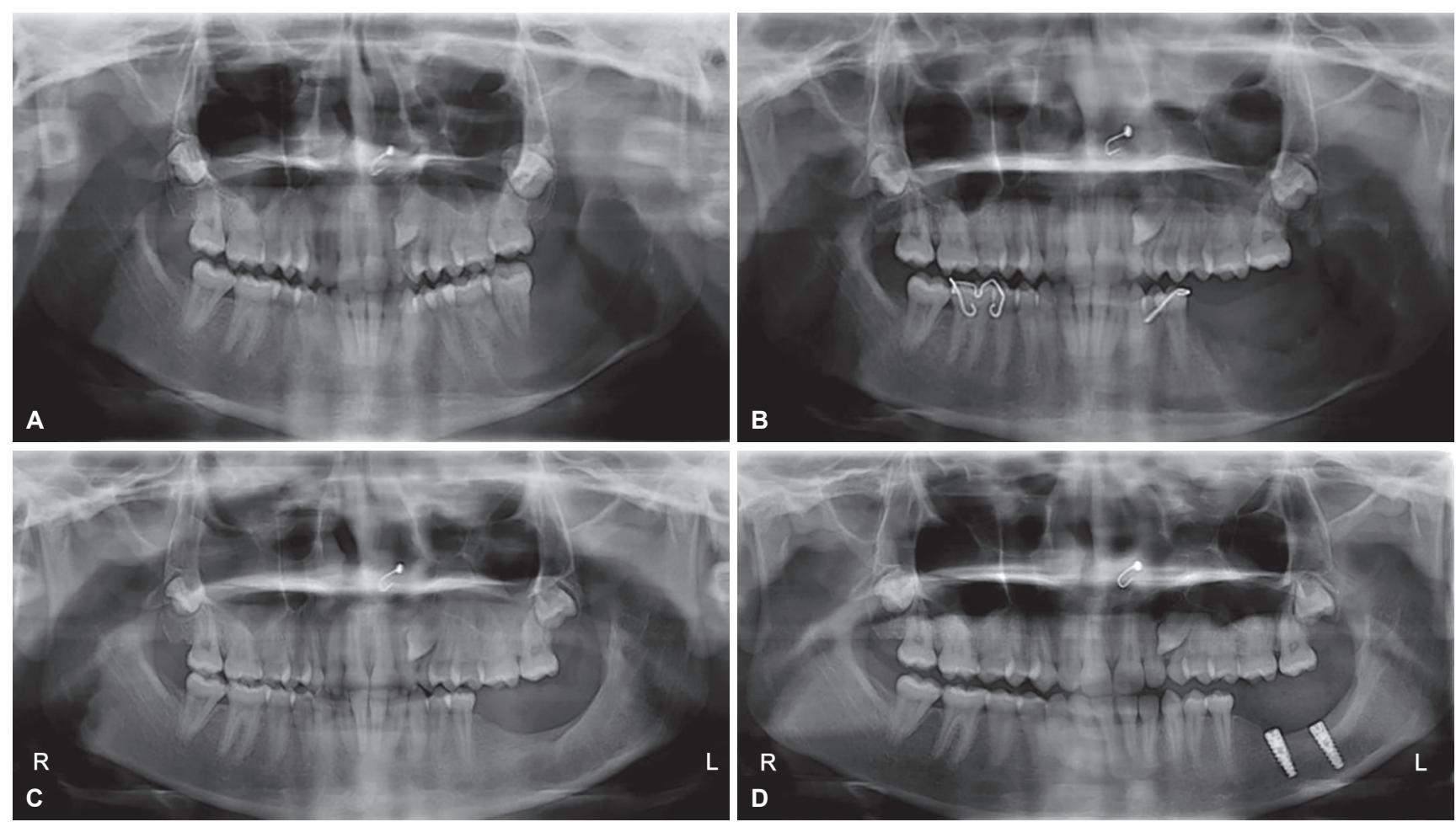

Figs 1A to D: (A) Radiographic appearance of lesion; (B) immediate postoperative; (C) postoperative 5 years; and (D) implant rehabilitation 

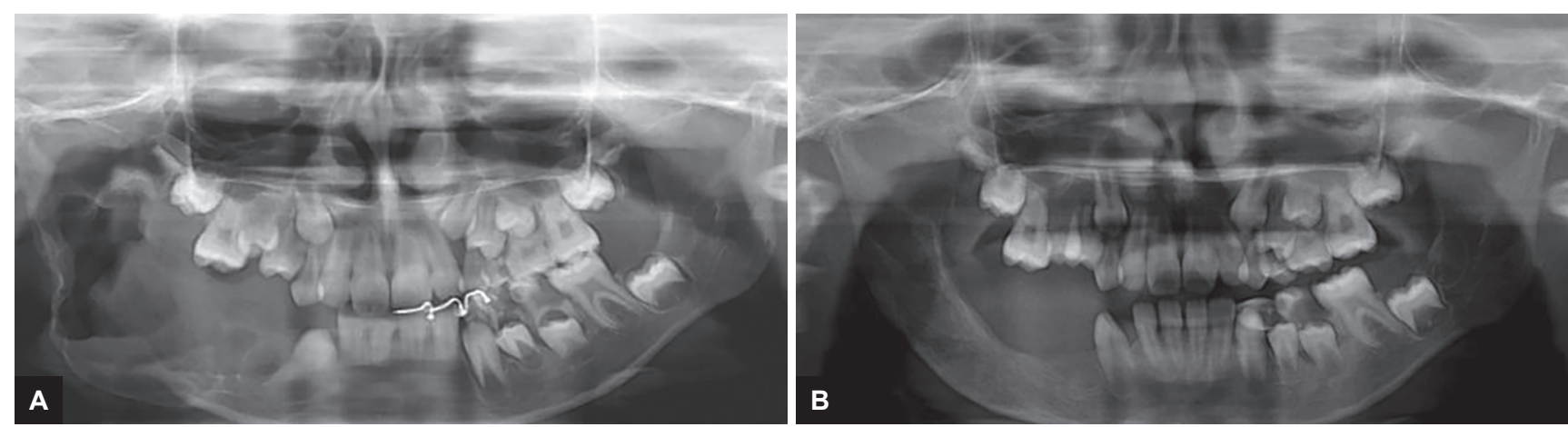

Figs 2A and B: (A) Radiographic appearance of lesion; and (B) postoperative 2 years

of all lesions was unilocular. In 15 cases, the size of the lesion exceeded $5 \mathrm{~cm}$ clinically, 14 patients had extraoral swelling, 1 patient with an infection, and 3 patients with neurosensory deficits. All the patients were treated using enucleation with peripheral ostectomy and application of Carnoy's solution for 5 minutes as a primary modality of choice postoperatively; patients with extensive lesion received an intermaxillary fixation for 5 to 6 weeks. Surgical obturator to obliterate the pathological defect was given in large defects which even allows for direct observation of the healing process and also imparts strength to the weakened jaw. The mean length of follow-up was 76 months since the last operation. As mentioned earlier, the eradication of the cyst is the principal goal in the treatment of OKCs. However, minimizing the recurrence and surgical morbidity is also crucial.

\section{DISCUSSION}

Treatment of UA and KCOT is controversial, and the treatment modality is influenced by factors, such as age, general health, anatomic locations, clinical behavior, and clinicoradiographic assessment of the lesion. Different treatment modalities available are enucleation, enucleation followed by use of Carnoy's solution, marsupialization followed by enucleation, marginal resection, and aggressive resection. ${ }^{7}$

Unicystic ameloblastoma unlike another variant of ameloblastoma is less aggressive and responds more favorably to conservative treatment modality. Supporters of the radical approach to the treatment of ameloblastoma present the view that these tumors are histologically benign in nature, less aggressive, and clinicopathologically lies between benign and malignant. ${ }^{8}$ Resection results in lowest recurrence rate $(3.6 \%)$ if adequate margins are removed. However, despite high success rate for resection, more conservative treatment to reduce morbidity and improve quality of life is considered. ${ }^{9}$

Owing to thin, friable wall of KCOT, it is difficult to enucleate the tumor in one piece, and with the presence of satellite cysts treatment should be to eliminate the possible cells left behind. Treatment is generally classified as conservative or aggressive. According to Blanas et al $^{10}$ enucleation of KCOT followed with an application of Carnoy's solution appears to be the least invasive procedure with the lowest recurrence rate. They reported that adding Carnoy's solution to the cyst cavity for 5 minutes after enucleation results in a recurrence rate comparable to that of resection without unnecessary aggressive surgery. ${ }^{10}$

This was also supported by Gosau et $\mathrm{al}^{11}$ who stated that enucleation combined with the application of Carnoy's solution reduced the recurrence rate compared with simple enucleation. The purpose of using Carnoy's solution is to provide a total elimination of epithelial remnants, which may cause recurrences. ${ }^{12}$

Care has to be taken while applying the solution to the tissue. First sterile gauze is placed, then gauze soaked in Carnoy's solution is placed in the pathological cavity for 5 minutes taking care to prevent any damage to the surrounding soft tissues. Irrigation with saline has to be performed after the removal of gauze.

The possible complications resulting from the use of Carnoy's solution include infection, dehiscence, bone sequestrum formation, and neuropathy. ${ }^{12}$ In contrast, another important study reported that when a proper protocol is followed, the chemical treatment of the nerve can be achieved without permanent functional damage. $^{13,14}$

Resection was earlier preferred, but it is associated with anatomic and functional morbidity. The advantage of Carnoy's solution and peripheral ostectomy is that it eliminates epithelial remnants and satellite cysts. ${ }^{15}$

These adjuncts, when used with enucleation, considerably decrease the recurrence rates. ${ }^{16}$ We recommend treating the bone defect around the cyst with Carnoy's solution and excising the overlying attached mucosa when cortices are perforated. In our study, no recurrence has been encountered till date using enucleation with peripheral ostectomy and application of Carnoy's solution as a primary modality of choice. 


\section{CONCLUSION}

The unilocular radiolucency of the jaws, such as UA and KCOT has a strong propensity for recurrences. Although resection of such lesions remains the gold standard, it also leads to a lifelong deformity associated with psychological effects, and irrespective of the reconstruction modality used, it is impossible to reconstruct the parabola architecture of the mandible.

On the contrary, enucleation and peripheral ostectomy with chemical cauterization helps to preserve the bone architecture along with maintaining the facial contour. Keeping in mind the possibility of a recurrence with enucleation and peripheral ostectomy with chemical cauterization, resection can always be reserved for a later date, if at all the need arises.

The use of a surgical obturator to obliterate the pathological defect allows direct observation of the healing process and also imparts strength to the weakened jaw. A minimum of 3 years follow-up is advised before these cases can be taken up for prosthetic rehabilitation even with the help of implants. Timely intervention and conservative surgical treatment followed by peripheral ostectomy and Carnoy's solution application improves outcome and other potential complications of surgery since this technique shows comparable results to other more aggressive techniques.

To conclude, we recommend it as treatment of choice for lesions, such as UA and KCOT as compared with surgical resection.

\section{REFERENCES}

1. Robinson L, Martinez MG. Unicystic ameloblastoma: a prognostic distinct entity. Cancer 1977 Nov;40(5):2278-2285.

2. Paikkatt VJ, Sreedharan S, Kannan VP. Unicystic ameloblastoma of the maxilla: a case report. J Indian Soc Pedod Prev Dent 2007 Apr-Jun;25(2):106-110.
3. Barnes, L.; Eveson, JW.; Reichart, P.; Sidransky, D. WHO classification of tumors: pathology and genetics head and neck tumors. Lyon: IARC Press; 2005. pp. 298-300.

4. Philipsen HP. Om keratocystes (kolesteatomer) i kaeberne. Tandlaegebl 1956;60:963-980.

5. Barnes, L. Surgical pathology of the head and neck. 2nd ed. Vol. 3. New York (NY): Marcel Dekker, Inc.; 2001.

6. Vered M, Buchner A, Dayan D, Shteif M, Laurian A. Solid variant of odontogenic keratocyst. J Oral Pathol Med 2004 Feb;33(2):125-128.

7. Sampson DE, Pogrel MA. Management of mandibular ameloblastoma: the clinical basis for a treatment algorithm. J Oral Maxillofac Surg 1999 Sep;579(9):1074-1077.

8. Ueno S, Mushimoto K, Shirasu R. Prognostic evaluation of ameloblastoma based on histologically and radio graphic typing. J Oral Maxillofac Surg 1989 Jan;47(1):11-15.

9. Kalaskar R, Unawane AS, Kalaskar RA, Pandilwar P. Conservative management of unicystic ameloblastoma in a young child: report of two cases. Contemp Clin Dent 2001 Oct-Dec;2(4):359-363.

10. Blanas N, Freund B, Schwartz M, Furst IM. Systematic review of the treatment and prognosis of the odontogenic keratocyst. Oral Surg Oral Med Oral Pathol Oral Radiol Endod 2000 Nov;90(5):553-558.

11. Gosau M, Draenert FG, Muller S, Frerich B, Bürgers R, Reichert TE, Driemei O. Two modifications in the treatment of keratocystic odontogenic tumors (KCOT) and the use of Carnoy's solution (CS) - a retrospective study lasting between 2 and 10 years. Clin Oral Investig 2010 Feb;14(1):27-34.

12. Stoelinga PJ, Bronkhorst FB. The incidence, multiple presentation and recurrence of aggressive cysts of the jaws. J Craniomaxillofac Surg 1988 May;16(4):184-195.

13. Frerich B, Cornelius $\mathrm{CP}$, Wiethölter H. Critical time of exposure of the rabbit inferior alveolar nerve to Carnoy's solution. J Oral Maxillofac Surg 1994 Jun;52(6):599-606.

14. Júnior OR, Borba AM, Ferreira CA, Júnior JG. Carnoy's solution over the inferior alveolar nerve as a complementary treatment for keratocystic odontogenic tumors. Rev Clín Pesq Odontol 2007 Sep-Dec;3(3):199-202.

15. Sivanmalai S, Kandhasamy K, Prabu N, Prince CN, Prabu CS. Carnoy's solution in the mangement of odontogenic keratocyst. J Pharm Bioallied Sci 2012 Aug;4(Suppl 2):S183-S185.

16. Pogrel MA. Enucleation plus additional physicochemical means to remove cyst remnants or daughter cysts in the peripheral bone. Curr Ther Oral Maxillofac Surg 2012. 